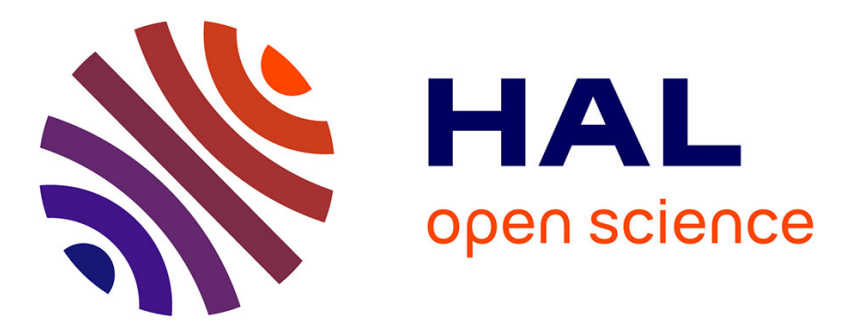

\title{
Energy storage due to strain-induced crystallization in natural rubber: The physical origin of the mechanical hysteresis \\ Jean-Benoit Le Cam
}

\section{To cite this version:}

Jean-Benoit Le Cam. Energy storage due to strain-induced crystallization in natural rubber: The physical origin of the mechanical hysteresis. Polymer, 2017, 127, pp.166-173. 10.1016/j.polymer.2017.08.059 . hal-01619285

HAL Id: hal-01619285

https://hal-univ-rennes1.archives-ouvertes.fr/hal-01619285

Submitted on 7 May 2020

HAL is a multi-disciplinary open access archive for the deposit and dissemination of scientific research documents, whether they are published or not. The documents may come from teaching and research institutions in France or abroad, or from public or private research centers.
L'archive ouverte pluridisciplinaire HAL, est destinée au dépôt et à la diffusion de documents scientifiques de niveau recherche, publiés ou non, émanant des établissements d'enseignement et de recherche français ou étrangers, des laboratoires publics ou privés.

\section{(c)(1)}

Distributed under a Creative Commons Attribution| 4.0 International License 


\title{
Energy storage due to strain-induced crystallization in natural rubber: the physical origin of the mechanical hysteresis
}

\author{
J.-B. Le Cam ${ }^{\mathrm{a}, \mathrm{b}, 1}$ \\ ${ }^{a}$ Université de Rennes 1, Institut de Physique UMR 6251 CNRS/Université de \\ Rennes 1, Campus de Beaulieu, Bât. 10B, 35042 Rennes Cedex, France. \\ ${ }^{\mathrm{b}}$ LC-DRIME, Joint Research Laboratory, Cooper Standard - Institut de Physique \\ UMR 6251, Campus de Beaulieu, Bât. 10B, 35042 Rennes Cedex, France.
}

\begin{abstract}
Strain-induced crystallization is classically assumed to be responsible for the hysteresis loop observed in the mechanical response of cis-1,4-polyisoprene. The aim of the present study is to investigate where does this energy go. Energy balances carried out using infrared thermography have shown that the hysteresis loop is due neither to intrinsic nor thermal dissipation, but is entirely used by the material to change its microstructure. Thus, significant changes in the internal energy accompany SIC. Experiments performed show that the mechanical energy brought to deform the material is stored elastically in the amorphous phase (chain alignment and accumulation of topological constraints in the crystallite vicinity) and is released with a different kinetics during crystallite melting. The demonstration that NR is able to store mechanical energy without converting it into heat is a realistic way to explain its extraordinary resistance to crack growth.
\end{abstract}

Key words: strain-induced crystallization, natural rubber, elastic energy stored, internal energy change.

\section{Introduction}

Mechanical properties of natural rubber (NR), cis-1,4-polyisoprene, are mainly related to Strain-Induced Crystallization (SIC) (Yijing et al., 2017). Especially, SIC enhances NR's resistance to crack growth (Lee and Donovan, 1987;

$\overline{1}$ Corresponding author jean-benoit.lecam@univ-rennes1.fr Fax : (+33) 223236111 
Lake, 1995) and fatigue (Cadwell et al., 1940; Beatty, 1964; Saintier, 2000; Le Cam et al., 2013). SIC in NR has been investigated extensively by means of X-ray diffraction technique since the pioneer work by Katz in 1925. A number of studies were dedicated to the crystallographic structure of NR (see for instance Bunn (1942) and Takahashi and Kumano (2004) for the monoclinic structure of crystal, and Immirzi et al. (2005) and Rajkumar et al. (2006) for the debate on the existence of orthorhombic cell). Several studies investigating the relationship between crystallinity and strain (Goppel and Arlman, 1949; Toki et al., 2000; Trabelsi et al., 2003a) have highlighted that crystallinity at a given strain is higher during unloading than during loading. Moreover, crystallite melting is complete at a lower stretch than that at which crystallization starts. This was attributed to a supercooling effect by Trabelsi et al. (2003a). However, numerous other phenomena accompanying SIC are still misunderstood, for instance the opposite effects of SIC on stress: on the one hand, SIC acts as network points, which increases the network chain density and leads to stress hardening Flory (1947); on the other hand, SIC induces stress relaxation of the amorphous phase (a plateau is observed in the strain-stress curve once SIC starts (Trabelsi et al., 2003a; Toki et al., 2000)). Moreover, as reported by Albouy and co-workers, SIC might develop at constant strain of the amorphous chains (Albouy et al., 2012, 2014). This raises several questions, among them: is such chain relaxation an exothermal phenomenon as observed in viscous materials? In other words, is there part of the mechanical energy brought converted into heat? Is the hysteresis loop due to SIC itself or to changes in the amorphous phase during crystallization?

Clark et al. (1940) experiments were the first to suggest that the mechanical hysteresis is closely related to SIC. To date, the idea that mechanical hysteresis is due to the kinetics difference between crystallization and crystallite melting has been widely disseminated, but no further information about the macromolecular origin of this mechanical energy dissipation has been provided. A recent study by Laghmach et al. on phase field modeling of SIC suggests that energy may be stored elastically in the vicinity of the crystal where topological constraints are concentrated (Laghmach et al., 2015), but to date no experimental evidence of this phenomenon has been provided.

In this paper, the contribution of the different phenomena involved in the formation of the mechanical hysteresis in NR is evaluated from energy balances. These energy balances are carried out using infrared thermography and the heat diffusion equation in the framework of irreversible thermodynamics processes. They enable us to determine first whether intrinsic dissipation is produced during the mechanical cycles and second to compare the strain power density with the heat power density at any time during the deformation process in order to detect, to quantify and to discuss any non-entropic effects.

Section 2 recalls the thermodynamic framework for energy balances. Section 
3 presents the results. Concluding remarks close the paper.

\section{Thermodynamic framework}

Rubber elasticity is mainly entropic. During stretching, polymer chains align, with less and less possible confirmations than in the relaxed state. Under adiabatic conditions, the kinetic theory due to Meyer and Ferri (1935) considers that the whole work done on the system by the external force is transferred into microscopic kinetic energy. This means that, contrary to steel, no potential energy would be stored by the rubber upon stretching. In this theory, rubber elasticity is only driven by change in configurational entropy. The internal energy is therefore constant during the deformation process at constant temperature. In such a context, applying the first law of thermodynamics leads to write that the changes in mechanical work $(\mathrm{dW})$ and in heat $(\mathrm{dQ})$ given out by the material on extension (which explains the negative sign) are equal:

$$
d W=-d Q
$$

This representation of rubber elasticity has been widely used to model rubber elasticity. Nevertheless, some deviations from this theory have been reported in the literature. For instance, the thermal response of vulcanized rubber is characterized by a slight cooling during stretching in the very low stretch range (typically for stretches inferior to 1.1), followed by a strong heating (Joule, 1857; Anthony et al., 1942; Pottier et al., 2009). Such thermo-sensitivity, is explained by preponderant effects of internal energy changes at low stretches. At higher stretches, the competition is in favor of the entropy changes, leading to a thermoelastic inversion. Several authors have measured the contribution of the internal energy to tension force for numerous rubber materials (Ciferri, 1961; Roe and Krigbaum, 1962; Allen et al., 1963; Shen, 1969; Boyce and Treloar, 1970; Allen et al., 1971; Price et al., 1969). The order of magnitude of the relative internal energy contribution to the force for rubbery polymers is 0.15 in a strain range of 50 to $300 \%$. The reader can refer to Treloar (1973) for further information. To account for this phenomenon, Chadwick (1974) proposed a modified entropic theory using a volumetric part in the strain energy density formulation that contains an energetic contribution to rubber elasticity. In this representation, the changes in internal energy on extension are associated with changes in volume only, in other words with the change in the Van der Waals forces between molecules (see Gee (1946)). This is an intermolecular internal energy. However, as discussed in Treloar (1973), another cause of change in the internal energy is the change in conformation of the polymer molecule itself, "arising from energy barriers to rotation about bonds within the single chain". This corresponds to finite intramolecular internal energy, which contributes to the stress, even under constant volume conditions. 
The need for an internal energy associated with the polymer chains conformation was highlighted by Flory and co-workers as soon as 1960 (Flory et al., 1960; Flory, 1961). This phenomenon occurs before SIC starts, while a part of the macromolecules becomes increasingly more aligned (this is a condition for their crystallization Rault et al. (2006a,b)), and during SIC. To finish, crosslinks and entanglements strongly affect rubber elasticity (Edwards and Vilgis, 1986; Doi, 1996). They induce additional topological constraints that may be of significant effects on the internal energy change. In this paper, energy balances enable us to evaluate the level and evolution of internal energy changes in natural rubber. For this purpose several continuum quantities are used. Each of them are presented in the following.

\subsection{Total strain energy density and hysteresis loop}

The total strain energy density $W_{\text {def }}$ is the energy brought mechanically to deform the material. It corresponds to the area under the load (unload) strainstress curve and is calculated as follows in case of uniaxial tensile conditions:

$$
W_{d e f}^{\text {load }}=\int_{\lambda_{\min }}^{\lambda_{\max }} \pi d \lambda \text { and } W_{d e f}^{\text {unload }}=\int_{\lambda_{\max }}^{\lambda_{\min }} \pi d \lambda
$$

where $\lambda$ is the stretch defined as the ratio between current and initial lengths. $\pi$ is the nominal stress tensor, defined as the force per unit of initial (undeformed) surface. If the material's behavior is purely elastic and if the test is carried out under adiabatic loading conditions, the mechanical response obtained during a load-unload cycle is such that no hysteresis loop forms $\left(W_{d e f}^{\text {load }}=W_{d e f}^{\text {unload }}\right)$. If a hysteresis loop forms, the mechanical energy dissipated $W_{\text {hyst }}$ over one cycle is determined as follows:

$$
W_{\text {hyst }}=W_{\text {def }}^{\text {load }}-W_{\text {def }}^{\text {unload }}
$$

The corresponding power density $P_{\text {hyst }}$ is obtained by dividing $W_{h y s t}$ by the cycle duration.

The strain power density $P_{\text {strain }}$ at any time during the deformation is defined as:

$$
P_{\text {strain }}=\pi \dot{\lambda}
$$

\subsection{Heat power density}

The heat power density is also named heat source (Samaca Martinez et al., 2013c) or thermal power (Holzapfel, 2000; Mott et al., 2016) in the literature. 
During the mechanical cycle, the material produces and absorbs heat. Under non adiabatic test conditions, corresponding temperature variations are influenced by heat diffusion effects. Therefore, determining the heat power density from temperature measurements requires taking into account heat diffusion effects. For this purpose, a simplified formulation of the heat diffusion equation is used, considering the heat power density field as homogeneous during uniaxial tensile loading (Chrysochoos, 1995):

$$
\rho C\left(\dot{\theta}+\frac{\theta}{\tau}\right)=s
$$

where $\rho$ is the density, $C$ is the specific heat, $\theta$ is the temperature variation, $\tau$ is a characteristic time that is identified from a natural return to ambient temperature. The right-hand side of Equation 5 represents the heat power density $s$ or (the rate of heat produced or absorbed by the material). It can be divided into two terms that differ in nature:

- the intrinsic dissipation $d_{1}$ : this positive quantity corresponds to the heat production due to mechanical irreversibilities during the deformation process, for instance viscosity or damage;

- the thermomechanical couplings $s_{t m c}$ : they correspond to the couplings between the temperature and the other state variables, and describe reversible deformation processes.

In unfilled natural rubber, volume changes are small (Le Cam and Toussaint, 2008; Chenal et al., 2007) and the specific heat does not vary significantly, even when crystallization occurs (Vogt, 1937; Boissonnas, 1939; Mayor and Boissonnas, 1948), therefore the product $\rho C$ can be assumed to be constant. As the heat produced and absorbed due to thermomechanical $s_{t m c}$ couplings is the same, the temporal integration of Equation 5 over one mechanical cycle provides the energy due to intrinsic dissipation $W_{\text {intrinsic }}$ :

$$
W_{\text {intrinsic }}=\int_{\text {cycle }}\left(s_{t m c}+d_{1}\right) d t=\int_{\text {cycle }} d_{1} d t
$$

The corresponding intrinsic dissipation $d_{1}$ over one cycle is obtained by dividing $W_{\text {intrinsic }}$ by the cycle duration. It should be noted that $d_{1}$ is a mean value and is possibly non constant during the mechanical cycle.

The energy corresponding to heat diffusion effects $W_{\text {thermal }}$ is defined by:

$$
W_{\text {thermal }}=\int_{\text {cycle }} \rho C\left(\frac{\theta}{\tau}\right) d t
$$




\subsection{Energy balances}

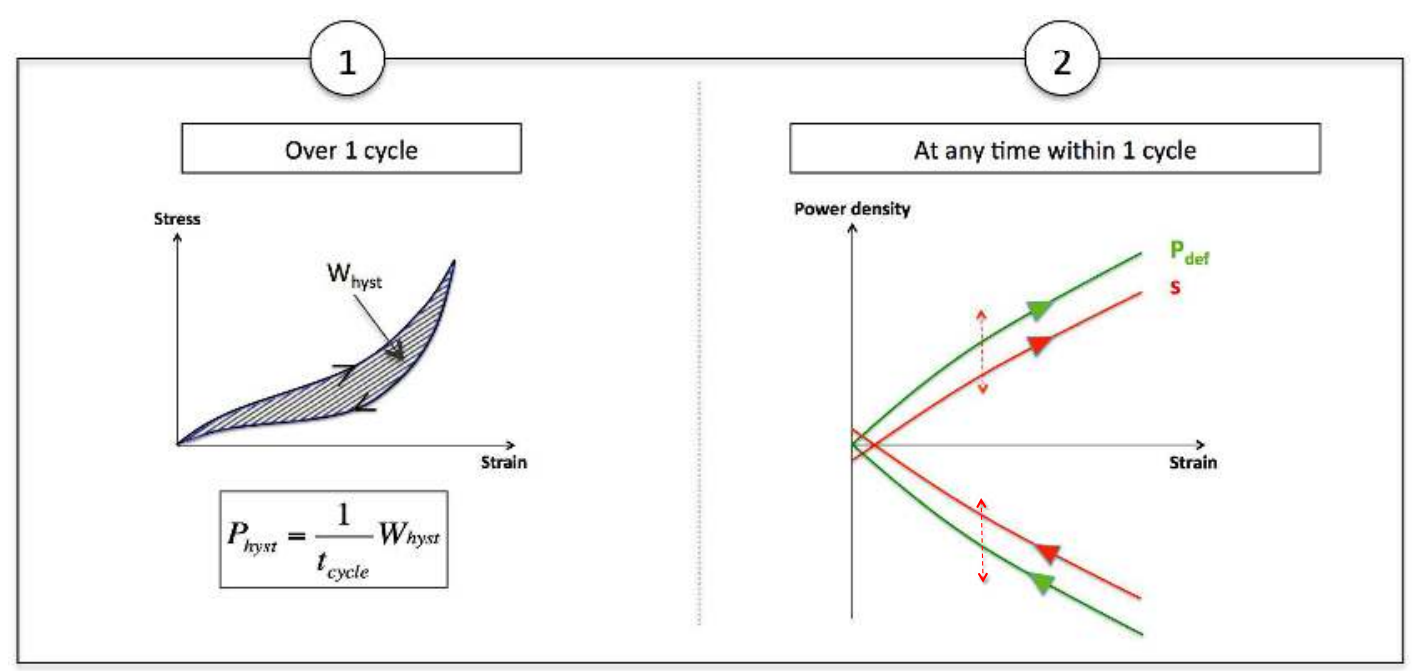

Fig. 1. Energy balance at two scales: the mechanical cycle (case \#1) and at any time within the mechanical cycle (case \#2)

Energy balances have to be carried out at two different scales. The first scale is a mechanical cycle (see Figure 1, schematic view \#1). The energy balance consists in comparing the energy density contained in the mechanical hysteresis $\left(W_{\text {hyst }}\right)$, more precisely the corresponding power density $\left(P_{\text {hyst }}\right)$, with the power densities associated with the following phenomena:

(a) intrinsic dissipation $\left(d_{1}\right)$ previously introduced,

(b) thermal dissipation $\left(d_{2}\right)$ (in non adiabatic test conditions). If heat is exchanged with the exterior of the specimen, a hysteresis loop in the stretchstress relationship forms, even for purely elastic materials,

(c) the part of the mechanical energy used by the material to change its microstructure $W_{\text {structure }}$, associated with changes in the internal energy.

Such analysis enables us to distinguish the part of the mechanical hysteresis converted into heat from that stored elastically and released by the material to change its microstructure.

The second scale at which energy balances are performed is any time (or 
stretch) during the mechanical cycle (see Figure 1, schematic view \#2). The energy balance consists in comparing the strain power density $\left(P_{\text {strain }}(\mathrm{t})\right)$ with the heat power density $(s(t))$. This comparison enables us to characterize the energetic contribution to the material's elasticity and to highlight kinetics effects in internal energy changes.

In these energy balances, the contribution of the thermal dissipation is negligible, this will be demonstrated in the next section for the test conditions of the present study.

\section{Experimental Section}

The material studied is an unfilled natural rubber (NR) supplied by the "Manufacture Française des pneumatiques Michelin". Its chemical composition is given in Table 1 . The compound was cured for $22 \mathrm{~min}$ at $150{ }^{\circ} \mathrm{C}$. The degree

Table 1

Chemical composition in parts per hundred rubber (phr).

\begin{tabular}{cc}
\hline Ingredient & Quantity \\
\hline Natural rubber NR & 100 \\
Carbon black & 0 \\
Antioxidant 6PPD & 1.9 \\
Stearic acid & 2 \\
Zinc oxide ZnO & 2.5 \\
Accelerator CBS & 1.6 \\
Sulfur solution $2 \mathrm{H}$ & 1.6 \\
\hline
\end{tabular}

of crosslinking is $6.510^{-5}$ mol.cm ${ }^{-3}$. In such NR formulation, SIC is observed in uniaxial tension starting from a stretch $\lambda_{c}$ of about 4. During unloading, crystallite melting is complete at a lower stretch $\lambda_{m}$ of about 3 (Toki et al., 2000; Trabelsi et al., 2003b; Le Cam and Toussaint, 2008). The specimen geometry is $5 \mathrm{~mm}$ wide, $10 \mathrm{~mm}$ high and $1.4 \mathrm{~mm}_{\text {thick }}{ }^{2}$. Mechanical loading was applied using a $50 \mathrm{~N}$ Instron 5543 testing machine. It corresponds to four sets of three cycles, for four increasing maximum stretch ratios: $\lambda_{1}=2$, $\lambda_{2}=5, \lambda_{3}=6$ and $\lambda_{4}=7.5$. $\lambda_{1}$ was chosen inferior to $\lambda_{c}, \lambda_{2}$ was superior but close to $\lambda_{c}, \lambda_{3}$ and $\lambda_{4}$ were superior to $\lambda_{c}\left(\lambda_{4}\right.$ was close to the stretch at

$\overline{2}$ In this paper, the mechanical tests performed were those reported in Samaca Martinez's PhD, which investigated the thermal and caloric signature of the main phenomena involved in rubber deformation (Samaca Martinez et al., 2013a,b,c; Samaca Martinez et al., 2014; Le Cam et al., 2015) 
failure). The signal shape chosen was triangular, to ensure a constant strain rate during loading and unloading. Tests were performed at a loading rate equal to $\pm 100 \mathrm{~mm} / \mathrm{min}$. The temperature was measured using a Cedip Jade III-MWIR infrared camera $(320 \times 240$ pixels, $3.5-5 \mu m)$.

\section{Results and discussion}

\subsection{Mechanical and thermal responses}

Figure 2(a) presents the mechanical response obtained for all the cycles performed in terms of the nominal stress in relation to the stretch. Figures 2(b) to (e) detail the mechanical response at the different maximum stretches applied. These figures show that whatever the maximum stretch applied, the mechanical cycles have no significant effect on the mechanical response, in the sense that no stress softening is observed. For cycles at $\lambda_{1}=2\left(<\lambda_{c}\right)$, a very small hysteresis loop is observed (see Fig. 2(a)). The corresponding power density $P_{\text {hyst }}$ is equal to $1.610^{3} \mathrm{~W} / \mathrm{m}^{3}$. For cycles at $\lambda_{2}=5\left(>\lambda_{c}\right)$, the area of the hysteresis loop significantly increases $\left(P_{\text {hyst }}=4.810^{3} \mathrm{~W} / \mathrm{m}^{3}\right.$, see Fig. $\left.2(\mathrm{~b})\right)$. It should be recalled that the stretch at which crystallization starts is about 4 . These results are in good agreement with those reported in the literature for unfilled natural rubber studied with X-ray diffraction: a significant hysteresis loop forms if SIC takes place in the material. Classically, the hysteresis loop is assumed to be due to the difference in the kinetics of crystallization and crystallite melting (Toki et al., 2000; Trabelsi et al., 2003b; Le Cam and Toussaint, 2008), but no further explanations on the mechanism leading to the mechanical energy lost have been provided. For cycles at $\lambda_{3}=6\left(>\lambda_{c}\right)$, the hysteresis loop is higher than for the previous stretches (see Fig. 2(c)). For cycles at $\lambda_{4}=7.5\left(>\lambda_{c}\right)$, a plateau is observed from $\lambda=6$ on, followed by a high stress increase (see Fig. 2(d)). The hysteresis loop is much higher than for the previous maximum stretch applied $\left(4.510^{4} \mathrm{~W} / \mathrm{m}^{3}\right.$ versus $\left.1.310^{4} \mathrm{~W} / \mathrm{m}^{3}\right)$. For higher stretches, a high increase in the nominal stress is observed: crystallites act as fillers and strongly reinforce the material stiffness. As assumed by Flory (1947) and highlighted experimentally by Toki et al. (2000) and Trabelsi et al.Trabelsi et al. (2003b), once crystallization occurs, relaxation is induced in the amorphous phase. The plateau observed is a manifestation of this relaxation. Moreover, according to Albouy and co-workers, SIC might develop at constant strain of the amorphous chains (Albouy et al., 2012, 2014). This raises several questions: is such chain relaxation an exothermal phenomenon as observed in viscous materials? In order words, is part of the mechanical energy brought converted into heat due to irreversibilities? Is the hysteresis loop due to SIC itself or to changes in the amorphous phase during crystallization? 


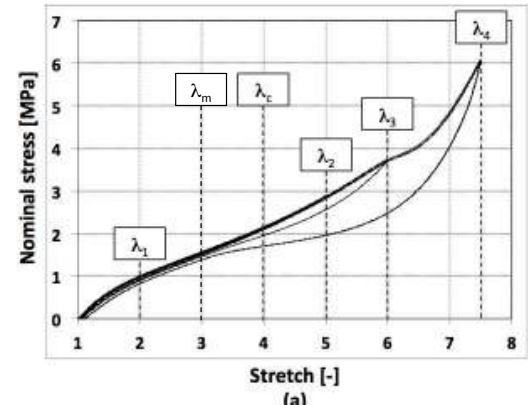

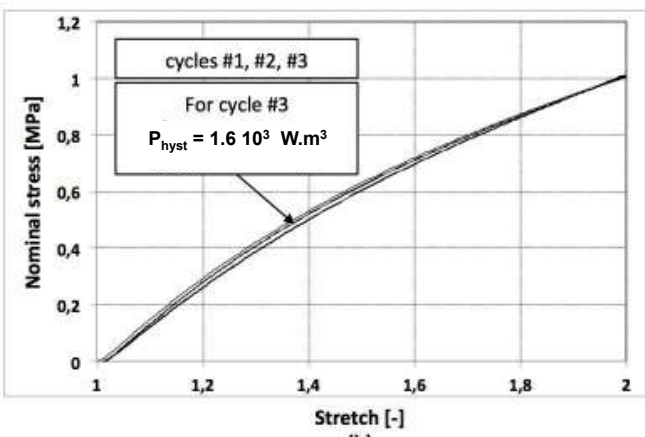

(b)

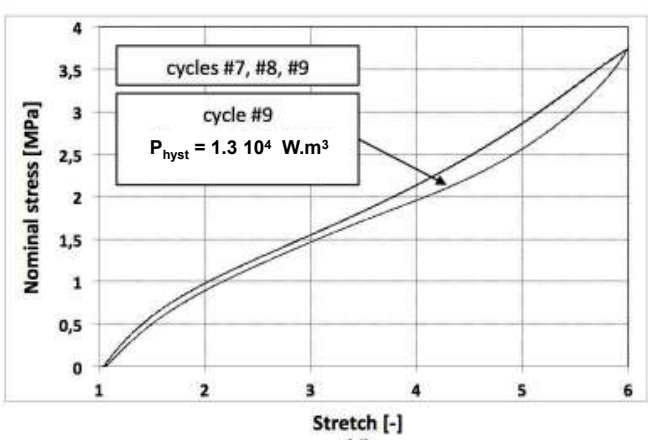

(d)
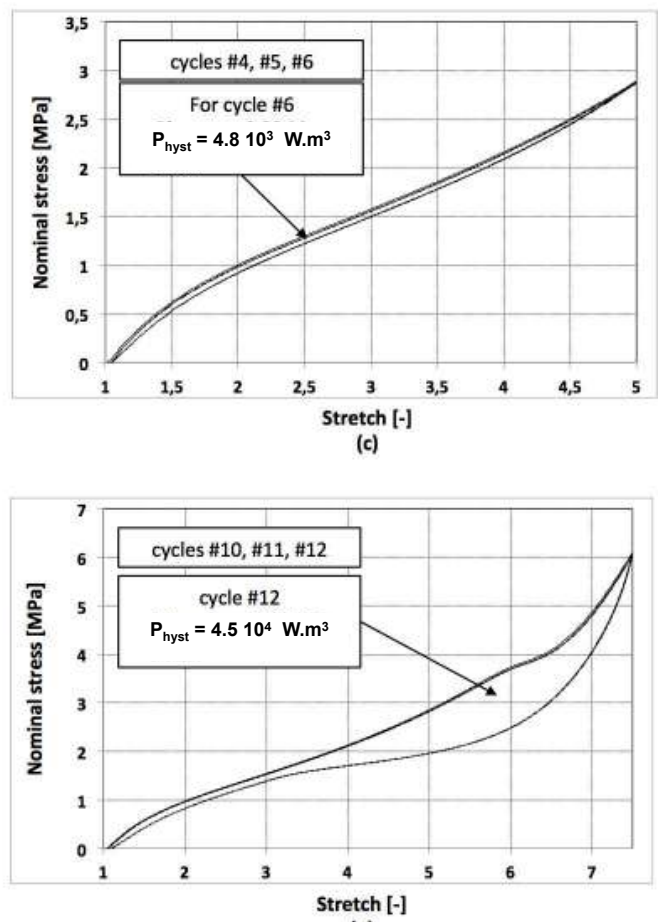

(e)

Fig. 2. Mechanical response

The thermal responses obtained during the mechanical cycles are depicted in Figure 3. First, results show that temperature variations are mainly due to entropic elasticity: they increase (decrease) when the stretch increases (decreases). Thermal accommodation is reached at the third cycle: temperature variation at the beginning and the end of the third cycles are the same. When SIC starts, a significant additional heat production is observed. It is highlighted by the dotted line (in red) plotted for the third (stabilized) cycles for $\lambda>\lambda_{c}$.

To discuss the effect of the thermal dissipation on the hysteresis loop area, two main arguments can be considered:

- Increasing the loading rate should change the value of the hysteresis loop area. This was not observed for tests performed at $\pm 300 \mathrm{~mm} / \mathrm{min}$ under the same conditions (see Fig. 4 in Samaca Martinez et al. (2013b)). This means that the effects of thermal dissipation on the hysteresis loop are negligible. 


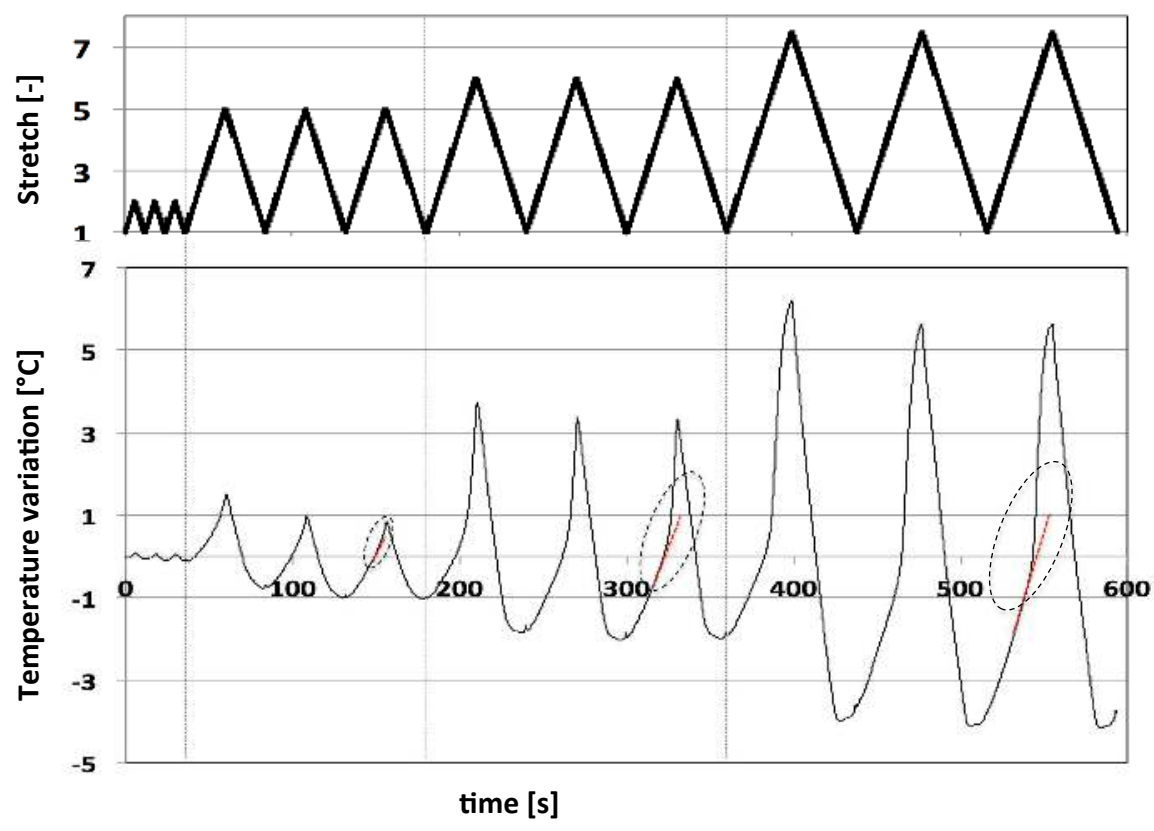

Fig. 3. Thermal response

- During cycles for which the same maximum stretches are applied, the mechanical hystereses are the same while temperature variations between the first and the third cycles are different.

For these reasons, the effect of thermal dissipation on the hysteresis loop area is not detectable and the mechanical energy dissipation can only be due to intrinsic dissipation and/or difference in the kinetics of microstructure changes. Distinguishing these two types of energy dissipation is of primal importance: the former is the signature of damage, the latter is not, which leads to very different consequences, especially for static and fatigue resistance to crack growth.

\subsubsection{Calorimetric response}

Figure 4 depicts the heat power density in relation to the stretch for a loading rate equal to $\pm 100 \mathrm{~mm} / \mathrm{min}$. It was established by using the simplified formulation of the heat diffusion equation (Eq. 5). Parameter $\tau$ was identified from natural return at ambient temperature for different stretches and written $\tau(\lambda)=40.48-3.25 \lambda$.

Several comments can be drawn from this figure. First, the heat source is positive during loading and increases with the stretch. During unloading, the heat source is negative. This highlights preponderant entropic effects observed in 


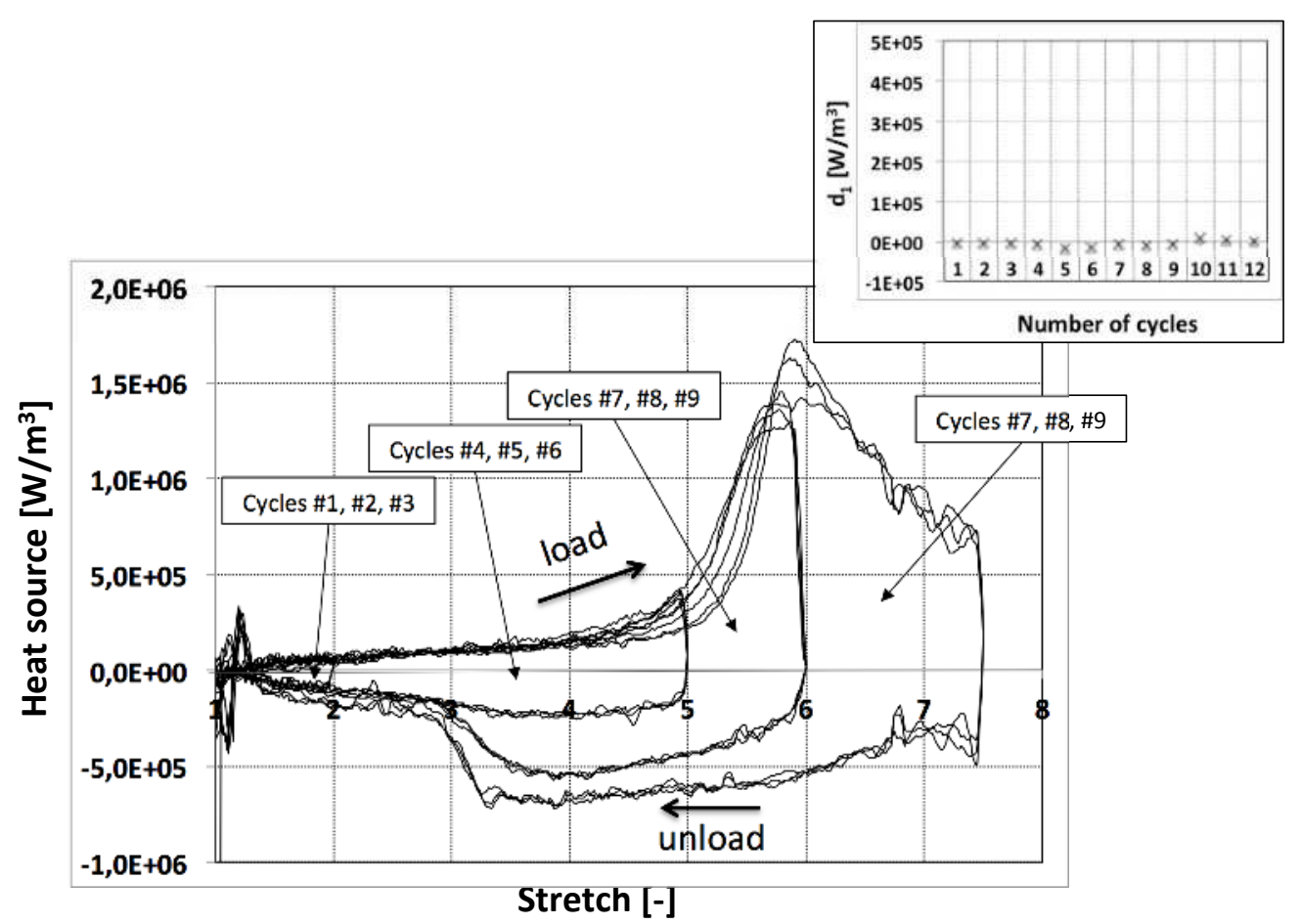

Fig. 4. Calorific response

the thermal response. Before SIC starts, the load-unload curves are symmetrical (see also Fig. 5(a)), meaning that the heat produced during the loading phase is equal to the heat source absorbed during the unloading phase. Thus, no intrinsic dissipation is detected.

Second, once SIC starts, the heat source evolution for loading and unloading are no longer symmetrical. This cannot be explained by entropic elasticity. During loading, the heat source evolves in a quasi-linear manner until a stretch close to 4 is reached. Dissymmetry occurs for stretches higher than 4 , the stretch level at which SIC starts. During unloading, the heat source rate first increases in absolute value until reaching a stretch equal to 4 , is constant until a stretch equal to 3.5 is reached, and then decreases. In order words, at a given stretch, heat absorption during unloading is lower than heat production during loading. Corresponding thermal measurements highlight that temperature is higher during unloading than during loading (see Fig. 3). As X-ray diffraction (crystallinity versus stretch curves) shows that crystallinity is higher during unloading than during loading (Huneau, 2011; Yijing et al., 2017 ) and that the dissolution of the crystalline order in NR is faster than its establishment after a strain step (Bruening et al., 2012), this demonstrates that crystallization continues during unloading. For each cycle, the energy 
corresponding to the intrinsic dissipation $W_{\text {intrinsic }}$ has been calculated by integrating the heat source. Intrinsic dissipation $d_{1}$ is obtained by dividing $W_{\text {intrinsic }}$ by the duration of the cycle considered. The values obtained, which are reported in the diagram in Figure 4, show that no intrinsic dissipation was detected whatever the mechanical cycle considered. This is consistent with the fact that no self-heating was observed in the thermal response. Consequently, the only explanation for the hysteresis loop is that the material stores energy elastically to change its microstructure, which involves a change in the internal energy. This is more precisely discussed in the next paragraph. Third, it is observed that the area of the hysteresis loop increases with the maximum stretch reached, meaning that the higher the crystallinity, the higher the area (mechanical energy) of the hysteresis loop.

Finally, for cycles at $\lambda>6$, the evolution of the heat source differs from that obtained for lower stretches. Indeed, during the loading phase, instead of increasing continuously, the heat source decreases from $\lambda=6$ on. This means that either heat due to crystallization continues to be produced (it remains positive), but at a lower rate, and/or that larger energetic effects take place (negative heat sources for positive stretch rates). The fact that the hysteresis loop area is larger than before $\left(4.510^{4} \mathrm{~W} / \mathrm{m}^{3}\right.$ versus $\left.1.310^{4} \mathrm{~W} / \mathrm{m}^{3}\right)$ pleads in favor of SIC.

\subsubsection{Elastic energy stored and released during deformation cycles}

As the mechanical hysteresis is due neither to thermal nor intrinsic dissipation, the mechanical energy involved in the hysteresis loop is used by the material to change its microstructure. This leads to elastic energy storage and thus change in the internal energy. To better characterize this effect and to highlight kinetics effects in the internal energy change, energy balances were carried out at any time during the deformation cycles, by comparing the strain power density $P_{\text {strain }}$ and the heat power density $s$. For instance, in case of purely entropic elasticity (see the kinetic theory due to Meyer and Ferri (1935)), the strain power density $P_{\text {strain }}$ is equal to the heat power density $s$ at any stretch. This type of energy balance has been successfully carried out in Mott et al. (2016) to investigate where does mechanical energy brought to a polyurethane material go. Results obtained by the authors showed that significant part of the mechanical energy brought is not converted into heat and is used by the material to change its microstructure. Figure 5 presents the evolution of $P_{\text {strain }}$ (in black) and $s$ (in red) for each set of maximum stretch.

For the first cycles at $\lambda_{1}=2<\lambda_{c}$ (see Fig. 4a), these powers are not equal $\left(P_{\text {strain }}>s\right)$, far from it. This highlights a strong non-entropic contribution to the rubber elasticity. Therefore, considering the previous discussions, the material stores elastic energy, which change the internal energy and affects 

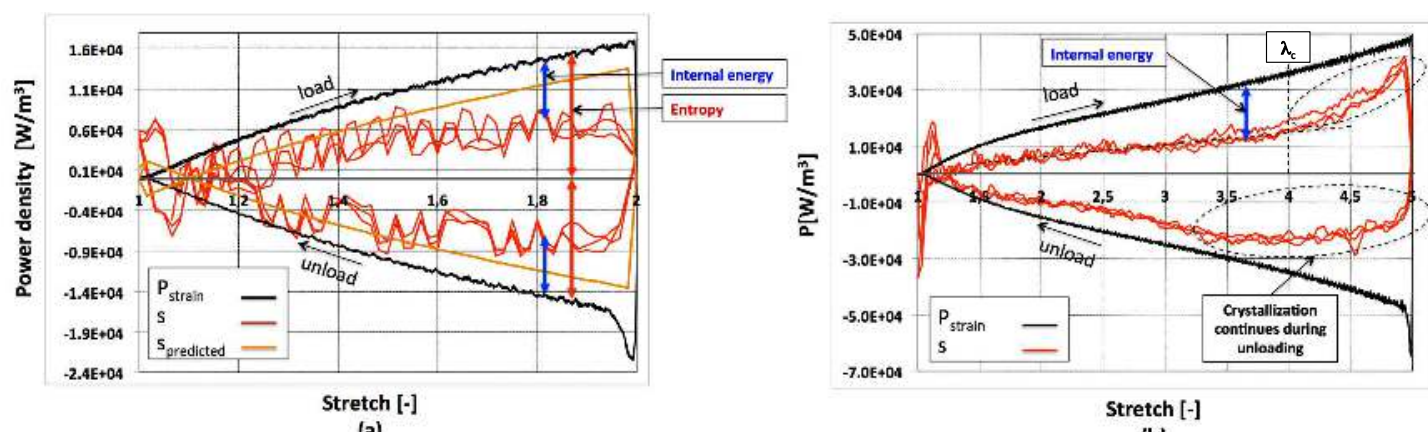

(b)

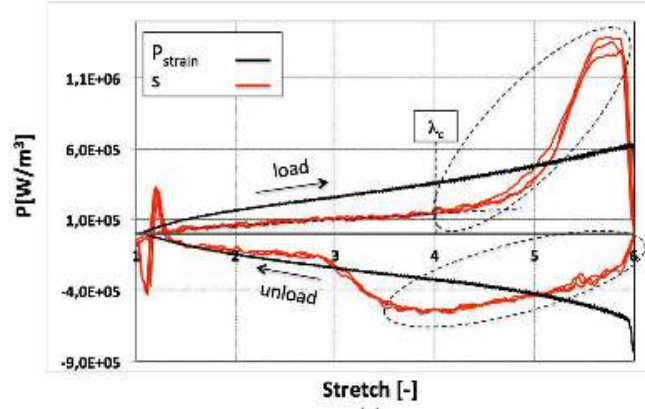

(c)

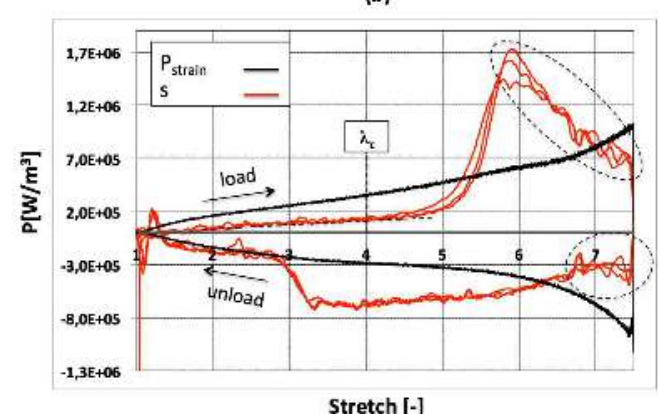

(d)

Fig. 5. evolution of the strain power and the heat power densities (a) $\lambda_{1}=2$ (a) $\lambda_{2}=5$ (a) $\lambda_{3}=6$ (a) $\lambda_{4}=7.5$

the calorific response. In this figure, a prediction of heat sources due to both entropy and intermolecular internal energy is plotted in orange. The results show that the model fits the stretch level at which thermoelastic inversion occurs and the heat sources until reaching a stretch of about 1.5. Second, from $\lambda=1.5$ on, the experimental heat sources are lower than predicted. This means that the area between the red and orange curves corresponds to additional energy stored by the material. As Treloar (1973) explained, a change in the conformation of polymer chains on extension leads to an additional change in the (intramolecular) internal energy, without volume variation, contrarily to intermolecular effects. The fact that a small hysteresis loop is observed for maximum stretches inferior to that at which SIC starts shows that chain alignment is a reversible processus, with no real difference in kinetics between loading and unloading. This is confirmed by the symmetrical evolution of the heat source between loading and unloading: the same amount of elastic energy is stored and released at a given stretch during loading and unloading.

When the stretch is superior to 2 and inferior to $\lambda_{c}$ (see Fig. 4b), the tendency observed during loading at the previous stretch is observed again: $P_{\text {strain }}>s$ and energy stored elastically increases. In this range of stretch the chain alignment, which is a detectable oriented amorphous phase before crystallization 
(see for instance the work by Toki et al. (2004)), has an increasing effect on the change in internal energy. It should be noted that this non-entropic contribution to rubber elasticity reaches more than $65 \%$ of what should be the entropic contribution in terms of heat power density at $\lambda=4$, i.e. before SIC starts. It is to note that topological constraints induced by entanglements and cross-links should also contribute to internal energy changes. Once SIC starts, the energy balance is more complicated due to the strong exothermal effect induced and several results have to be put into perspective. We have shown above that chain relaxation as well as chain alignment occur without producing intrinsic dissipation. Moreover, Laghmach et al. have recently suggested in a modeling approach of SIC that topological constraints due to entanglements and cross-links are pushed away from the crystal phase to allow further crystallization (Laghmach et al., 2015). To the authors, the accumulation of these topological constraints in the vicinity of the crystal interface may store elastic energy in the amorphous phase. The energy balances carried out in the present paper provide the experimental evidence that SIC stores elastic energy, which contributes significantly to change the internal energy: during loading first, but also during unloading, since SIC continues. Elastic energy is released with a different kinetics when crystallites melt, but also as soon as the beginning of the unloading phase, which explains the strong decrease in the stress. The assumption that topological constraint zones close to the crystallites store energy in the amorphous phase is therefore realistic. From a certain crystallinity (typically obtained at $\lambda=6$ ), the accumulation of topological constraints limits the crystallite growth, which decreases the rate of crystallization and increase the change in internal energy. For these two reasons, the heat power density rate decreases.

\section{Conclusion}

Mechanical energy dissipated by natural rubber, which corresponds to the mechanical hysteresis area, is due neither to intrinsic nor thermal dissipation, meaning that no mechanical energy brought to the material during cyclic loadings is converted into heat. This result has numerous consequences: natural rubber (NR) does not exhibit any viscosity, even when crystallizing, and the energy dissipated is entirely used by the material to change its microstructure. Energy balances performed at any time during the deformation cycles show that NR elasticity exhibits cyclic variation of its internal energy. For stretches inferior to that at which SIC starts, the variation is symmetrical between loading and unloading and is mainly due interactions between macromolecules, cross-links and entanglements. From $\lambda=1.5$ on, and additional internal energy change is observed that could be attributed to the increasing effect of chain alignment. The fact that the kinetics of internal energy change 
is the same between loading and unloading explains why no significant mechanical hysteresis forms. This non-entropic contribution to rubber elasticity represents more than half of the entropic contribution at stretches inferior that at which crystallization starts. This demonstrates that the calorific and thermal response of natural rubber can not be determined accurately without considering such non-entropic effects. When SIC occurs, additional topological constraints are induced in the amorphous phase close to the crystallites, they store energy and change the internal energy of the system. As SIC and crystallite melting have different kinetics, the cyclic internal energy change is dissymmetric. Thus, crystallization/crystallite melting processus stores and releases mechanical energy with different rates, which forms the hysteresis loop. More than SIC itself as a thermodynamical processus, the explanation of the hysteresis loop formation lies in the kinetics of microstructural changes, and therefore of internal energy change, induced by SIC in the amorphous phase. The demonstration that NR is able to store elastically part of the mechanical energy brought to it without converting it into heat is a realistic way to explain its extraordinary resistance to crack growth.

\section{Acknowledgements}

The author acknowledges the "Manufacture Française des pneumatiques Michelin" for supporting this study. The author thanks J.R. Samaca Martinez, many results reported in this paper were obtained during his $\mathrm{PhD}$ Thesis. X. Balandraud, E. Toussaint, J. Caillard and D. Berghezan are acknowledged for the fruitful discussions.

\section{References}

Albouy, P.-A., Guillier, G., Petermann, D., Vieyres, A., Sanseau, O., and Sotta, P. (2012). A stroboscopic X-ray apparatus for the study of the kinetics of strain-induced crystallization in natural rubber. Polymer, 53, 3313-3324.

Albouy, P.-A., Vieyres, A., Perez-Aparicio, R., Sanseau, O., and Sotta, P. (2014). The impact of strain-induced crystallization on strain during mechanical cycling of cross-linked natural rubber. Polymer, 55, 4022-4031.

Allen, G., Bianchi, U., and Price, C. (1963). Trans. Faraday Soc., 59, 24932502.

Allen, G., Kirkham, M., Padget, J., and Price, C. (1971). Trans. Faraday Soc., 67, 1278-1292.

Anthony, R. L., Caston, R. H., and Guth, E. (1942). Equations of state for naturals and synthetic rubber like materials: unaccelerated natural soft rubber. Journal of Physical Chemistry, 46, 826. 
Beatty, J. R. (1964). Fatigue of rubber. Rubber Chemistry and Technology, 37, 1341-1364.

Boissonnas, C. (1939). Ind. Eng. Chem., 31, 761.

Boyce, P. and Treloar, L. (1970). Polymer, 11, 21-30.

Bruening, K., Schneider, K., Roth, S. V., and Heinrich, G. (2012). Kinetics of strain-induced crystallization in natural rubber studied by waxd: Dynamic and impact tensile experiments. Macromolecules, 45, 7914-7919.

Bunn, C. (1942). . Proc. R. Soc. London, Ser. 1, 180, 40.

Cadwell, S. M., Merril, R. A., Sloman, C. M., and Yost, F. L. (1940). Dynamic fatigue life of rubber. Industrial and Engineering Chemistry (reprinted in Rubber Chem. and Tech. 1940;13:304-315), 12, 19-23.

Chadwick, P. (1974). Thermo-mechanics of rubberlike materials. Philosophical Transactions of the Royal Society London, A276, 371.

Chenal, J.-M., Gauthier, C., Chazeau, L., Guy, L., and Bomal, Y. (2007). Parameters governing strain induced crystallization in filled natural rubber. Polymer, 48, 6893-6901.

Chrysochoos, A. (1995). Analyse du comportement des matériaux par thermographie infra rouge. In Colloque Photomécanique, volume 95, pages 201-211.

Ciferri, A. (1961). Makromol. Chem., 43, 152-155.

Clark, G. L., Kabler, M., Blaker, E., and Ball, J. M. (1940). Hysteresis in crystallization of stretched vulcanized rubber from x-ray data. Industrial and Engineering Chemistry, 32, 1474-1477.

Doi, M. (1996). Introduction to Polymer Physics. Oxford Science Publications, Oxford.

Edwards, S. F. and Vilgis, T. A. (1986). The effect of entanglements in rubber elasticity. Polymer, 27, 483-492.

Flory, P. J. (1947). Thermodynamics of crystallization in high polymers. i. crystallization induced by stretching. The Journal of Chemical Physics, 15, 397-408.

Flory, P. J. (1961). Thermodynamics relation for high elastic materials. Transactions of the Faraday Society, 57, 829-838.

Flory, P. J., Ciferri, A., and Hoeve, C. A. J. (1960). The thermodynamic analysis of thermoelastic measurements on high elastic materials. Journal of Polymer Science, 45, 235-236.

Gee, G. (1946). Trans. Faraday. Soc., 42, 585.

Goppel, J. and Arlman, J. (1949). . Appl. Sci. Res. Sect. A, 1, 462.

Holzapfel, G.-A. (2000). Non linear solid mechanics - a continuum approach for engineering. Wiley.

Huneau, B. (2011). Strain-induced crystallization of natural rubber: a review of X-ray diffraction investigations. Rubber Chemistry And Technology, 84, $425-452$.

Immirzi, A., Tedesco, C., Monaco, G., and Tonelli, A. (2005). . Macromolecules, 38, 1223.

Joule, J. P. (1857). On some thermodynamic properties of solids. Phil Mag 4th, 14, 227. 
Katz, J. (1925). Naturw, 4, 169.

Laghmach, R., Candau, N., Chazeau, L., Munch, E., and Biben, T. (2015). Phase field modelling of strain induced crystal growth in an elastic matrix. The Journal of Chemical Physics, 142, 244905.

Lake, G. J. (1995). Fatigue and fracture of elastomers. Rubber Chemistry and Technology, 68, 435-460.

Le Cam, J.-B. and Toussaint, E. (2008). Volume variation in stretched natural rubber: competition between cavitation and stress-induced crystallization. Macromolecules, 41, 7579-7583.

Le Cam, J.-B., Huneau, B., and Verron, E. (2013). Fatigue damage in carbon black filled natural rubber under uni- and multiaxial loading conditions. International Journal of Fatigue, 52, 82 - 94.

Le Cam, J.-B., Samaca Martinez, J. R., Balandraud, X., Toussaint, E., and Caillard, J. (2015). Thermomechanical Analysis of the Singular Behavior of Rubber: Entropic Elasticity, Reinforcement by Fillers, Strain-Induced Crystallization and the Mullins Effect. Experimental Mechanics, 55, 771782.

Lee, D. J. and Donovan, J. A. (1987). Microstructural changes in the crack tip region of carbon-black-filled natural rubber. Rubber Chemistry and Technology, 60, 910-923.

Mayor, A. R. and Boissonnas, C.-G. (1948). Variation de la chaleur spécifique du caoutchouc en fonction de l'allongement. Helvetica Chimica Acta, 31, $1514-1532$.

Meyer, K. H. and Ferri, C. (1935). Sur l'élasticité du caoutchouc. Helvetica Chimica Acta, 18, 570-589.

Mott, P., Giller, C., Fragiadakis, D., Rosenberg, D., and Roland, C. (2016). Deformation of polyurea: Where does the energy go? Polymer, 105, $227-$ 233.

Pottier, T., Moutrille, M.-P., Le Cam, J.-B., Balandraud, X., and Grédiac, M. (2009). Study on the use of motion compensation technique to determine heat sources. application to large deformations on cracked rubber specimens. Experimental Mechanics, 49, 561-574.

Price, C., Padget, J., Kirkham, M., and Allen, G. (1969). Polymer, 10, 573578.

Rajkumar, G., Squire, J., and Arnott, S. (2006). . Macromolecules, 39, 7004.

Rault, J., Marchal, J., Judeinstein, P., and Albouy, P.-A. (2006a). Macromolecules, 39, 8356-8368.

Rault, J., Marchal, J., Judeinstein, P., and Albouy, P.-A. (2006b). Eur. Phys. J. E.: Soft Matter Biol. Phys., 21, 243.

Roe, R. and Krigbaum, W. (1962). Polymer Sci., 61, 167-183.

Saintier, N. (2000). Prévisions de la durée de vie en fatigue du NR, sous chargement multiaxial. Thèse de doctorat, École Nationale Supérieure des Mines de Paris.

Samaca Martinez, J. R., Le Cam, J.-B., Balandraud, X., Toussaint, E., and Caillard, J. (2013a). Filler effects on the thermomechanical response of 
stretched rubbers. Polymer testing, 32, 835 - 841.

Samaca Martinez, J. R., Le Cam, J.-B., Balandraud, X., Toussaint, E., and Caillard, J. (2013b). Thermal and calorimetric effects accompanying the deformation of natural rubber. part 1: Thermal characterization. Polymer, 54, $2717-2726$.

Samaca Martinez, J. R., Le Cam, J.-B., Balandraud, X., Toussaint, E., and Caillard, J. (2013c). Thermal and calorimetric effects accompanying the deformation of natural rubber. part 2: quantitative calorimetric analysis. Polymer, 54, 2727 - 2736.

Samaca Martinez, J. R., Le Cam, J.-B., Balandraud, X., Toussaint, E., and Caillard, J. (2014). New elements concerning the Mullins effect: A thermomechanical analysis. European Polymer Journal, 55, 98-107.

Shen, M. (1969). Macromolecules, 2, 358-364.

Takahashi, Y. and Kumano, T. (2004). . Macromolecules, 37, 4860.

Toki, S., Fujimaki, T., and Okuyama, M. (2000). Strain-induced crystallization of natural rubber as detected real-time by wide-angle x-ray diffraction technique. Polymer, 41, 5423-5429.

Toki, S., Sics, I., Ran, S., Liu, L., Hsiao, B., Murakami, S., Tosaka, M., Kohjiya, S., Poompradub, S., Ikeda, Y., and Tsou, A. (2004). Rubber Chemistry and Technology, 42, 956-964.

Trabelsi, S., Albouy, P., and Rault, J. (2003a). Crystallization and melting processes in vulcanized stretched natural rubber. Macromolecules, 36, 76247639 .

Trabelsi, S., Albouy, P.-A., and Rault, J. (2003b). Effective local deformation in stretched filled rubber. Macromolecules, 36, 9093-9099.

Treloar, L. R. G. (1973). The elasticity and related properties of rubbers. Reports on Progress in Physics, 36, 755.

Vogt, W. (1937). The Chemistry and Technology of Rubber. Reinhold Publ. N.-Y.

Yijing, N., Zhouzhou, G., Ya, W., Tongfan, H., and Zhiping, Z. (2017). Features of strain-induced crystallization of natural rubber revealed by experiments and simulations. Polymer Journal, 49, 309-317. 